\title{
Retreatment of a Chronically Infected Mandibular Molar with Missed Middle Mesial Canal: A Case Report
}

\author{
Abraham Ashwin Eugine ${ }^{1}$, Sangeetha Saji ${ }^{2}$, K Radhakrishnan Nair ${ }^{3}$, Praveena Geetha ${ }^{4}$, Sreepooja CS $^{5}$
}

\begin{abstract}
Aim and background: A major cause of the failure of root canal therapy is the inability to locate and treat all the canals of the root canal system. Magnification and ultrasonics are valuable aids in negotiating missed canals. In case of root canal failures with long-standing lesions, a combination of non-surgical retreatment followed by a surgical approach would be more beneficial.

Case description: This case report describes the management of a mandibular molar with a missed middle mesial canal. The canal was negotiated with the help of magnification and ultrasonics. Since it was a long-standing lesion, a surgical intervention was done after the completion of non-surgical retreatment.

Conclusion: Untreated root canals may have a direct impact on the prognosis of root canal treatment resulting in chronic periapical infection. Development in techniques and armamentarium has led to successful identification and instrumentation of missed canals. A surgical approach for a long-standing lesion ensures complete healing.

Keywords: Magnification, Middle mesial canal, Retreatment, Ultrasonics.

Conservative Dentistry and Endodontic Journal (2021): 10.5005/jp-journals-10048-0069
\end{abstract}

\section{BACKGROUND}

Proper disinfection of the root canal system is an important factor influencing the outcome of endodontic treatment. Various factors such as persistent bacterial infection, inadequate root filling, untreated canals, improper coronal seal/restoration, and procedural errors might result in persistent apical periodontitis. Missed canals have a negative impact on the root canal treatment prognosis. The prevalence of missed canals in mandibular first molars was found to be $11.2 \%$.

Conventional radiography because of its two-dimensional nature presents limitations while searching for missed canals. With the advent of cone-beam computed tomography (CBCT), it has been able to visualize the pulpal anatomy by providing a more accurate three-dimensional representation. Cone-beam computed tomography helps in the determination of the lesion size, its extent, and identification of missed canals as well. Among the various aids used in the detection of missed canals, magnification plays an important role. The dental operating microscope (DOM) provides the clinician with superior lighting and visibility which improves the predictability of the procedures. Ultrasonics is another valuable tool in the identification of missed canals. Troughing with ultrasonic tips helps in removing the calcified layer above the canal orifices.

Cultures of infected and previously root-filled teeth produce very few or even a single species of microorganism, predominantly gram-positive, aerobic. A commonly isolated species is Enterococcus faecalis, which is resistant to canal disinfecting regimens. ${ }^{2}$ So, our disinfection protocol should be aimed at eradicating these microorganisms.

When a treated tooth is asymptomatic and a persisting periapical lesion is noted on follow-up radiographs, convincing the patient about the condition and the treatment options becomes a challenging task. The choice of non-surgical retreatment vs apical surgery becomes the focus of the decision in most instances. In the case of chronic lesions, better outcomes are obtained by combining both non-surgical and surgical approaches. This report describes a
${ }^{1-5}$ Department of Conservative Dentistry and Endodontics, Azeezia College of Dental Sciences and Research, Kollam, Kerala, India

Corresponding Author: Abraham Ashwin Eugine, Department of Conservative Dentistry and Endodontics, Azeezia College of Dental Sciences and Research, Kollam, Kerala, India, Phone: +91 9656929584, e-mail: drashwin.eugine@gmail.com

How to cite this article: Eugine AA, Saji S, Radhakrishnan Nair K, et al. Retreatment of a Chronically Infected Mandibular Molar with Missed Middle Mesial Canal: A Case Report. Cons Dent Endod J 2021;6(1): 14-17.

Source of support: Nil

Conflict of interest: None

case of retreatment of a mandibular molar with the missed middle mesial canal.

\section{Case Description}

A 20-year-old female reported to the department with the complaint of pain in relation to the upper left premolar for 2 weeks. On clinical examination, there was a deep class 1 carious lesion in relation to 25 and the tooth was tender on percussion. It was a case of symptomatic apical periodontitis. The root canal treatment of 25 was completed on the second appointment. After completion, she wanted to get a crown done for another root canal treated posterior tooth. Root canal treatment of 36 was done 5 years ago following a history of swelling. Now the patient is asymptomatic. Before proceeding with the definitive restoration, a periapical radiograph was made to assess the status of the root canal-treated tooth. Radiographic examination revealed an obturated tooth with a well-defined oval-shaped radiolucency in relation to 36 (Fig. 1).

To evaluate the size of the lesion, its extension and to rule out any missed canals, a CBCT was taken. Coronal view of CBCT revealed a large, well-defined corticated radiolucency that extends from 
the mesial root surface of 35 up to the distal surface of the mesial root of 37. The lesion measured $25.0 \mathrm{~mm}$ mesiodistally and 10.7 $\mathrm{mm}$ superoinferiorly. The lesion caused resorption of mesial roots of 36 and also displaced inferior alveolar nerve (IAN) inferiorly. No obliteration of buccal and lingual cortical plates was seen. Four canals could be appreciated in the axial view of $C B C T$ (MB, ML, middle mesial, and distal). Endodontic filling material was seen in $\mathrm{MB}, \mathrm{ML}$, and distal canals. There was a missed middle mesial canal which was left unfilled (Fig. 2).

The overall radiographic impression was of a benign cystic lesion with respect to the left mandibular body. Differential diagnosis was a periapical cyst, keratocystic odontogenic tumor (KCOT). The vitality status of the adjacent teeth (35 and 37) was checked, which was found to be vital. There were no pockets as well as mobility of the associated teeth (35-37). Since the tooth was asymptomatic, the condition and the treatment options were explained to the patient. After obtaining the consent, retreatment of 36 followed by the surgical intervention has opted as the treatment plan.

Under local anesthesia and rubber dam isolation, the composite restoration was removed and the pulp chamber was accessed. Gutta-percha was softened using a solvent and initial GP removal was done using Hedstrom files, following which rotary retreatment

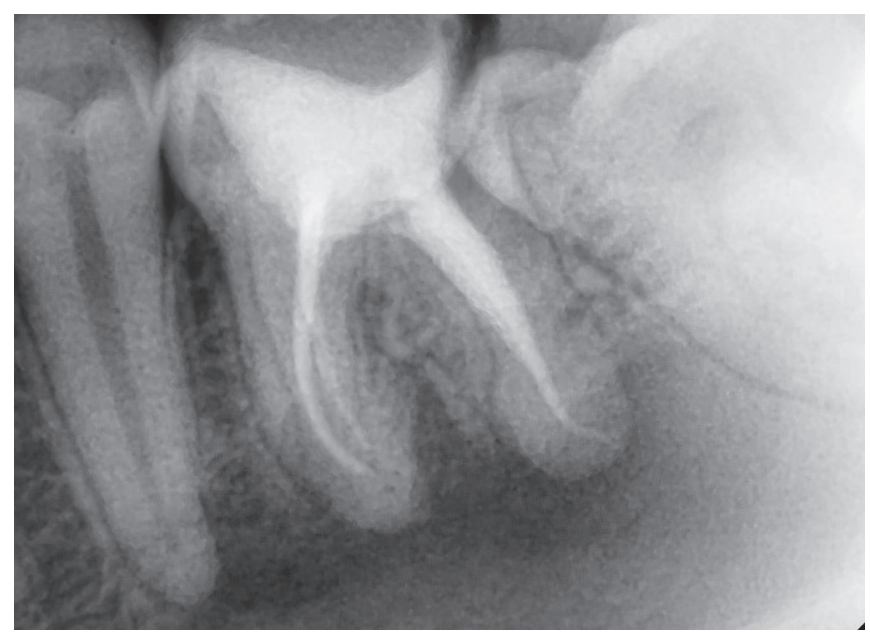

Fig. 1: Periapical radiograph shows the extent of the lesion

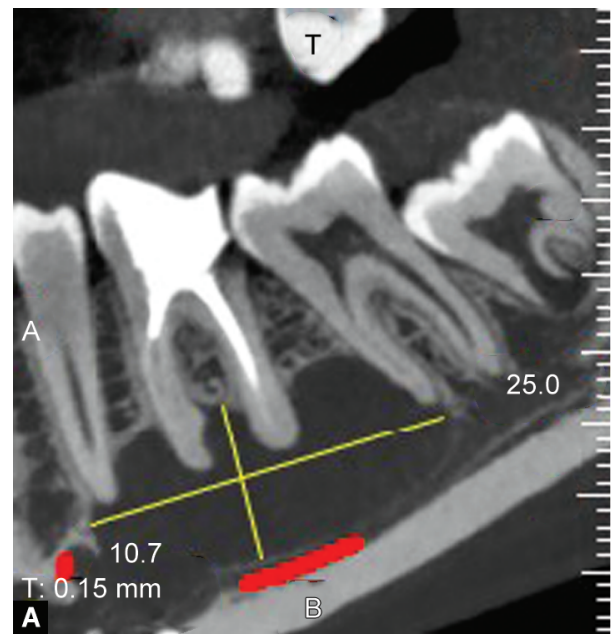

Figs 2A and B: (A) CBCT images: (A) Sagittal view; (B) Axial view files were employed. To locate the missed, middle mesial canal initial troughing was done using ultrasonic tips (P4D tip) following which a DG 16 explorer was used to locate the depression of the canal. After negotiation of the canals using 08 and 10 size $k$ files, orifice enlargement and working length determination were done. The procedures were performed under a DOM (Figs 3 and 4).

During biomechanical preparation, sodium hypochlorite (3\%) was used as an irrigant and calcium hydroxide dressing was given as intracanal medicament in-between appointments. After saline irrigation, chlorhexidine (2\%) was used as a final irrigant, the master cone was selected and obturation was done using the cold lateral condensation method (Fig. 5). Postendodontic restoration was done using composite.

An appointment was given for the patient for periapical surgery after the postendodontic restoration. Under local anesthesia, a crevicular incision was placed extending from the mesial aspect of 34 up to the distal aspect of 38 and a vertical releasing incision placed with respect to the mesial aspect of 34 and distal aspect of 38. A full-thickness mucoperiosteal flap was raised. The lesion was identified and a bone window was created. Peripheral ostectomy and complete enucleation of the lesion were done. The specimen was sent for histopathological examination. Bone graft was mixed with PRF and placed on the site. The flap was reapproximated and sutured using 3-0 vicryl sutures (Fig. 6). The histopathological report came out to be as periapical granuloma. The patient was reviewed after 1 week. Healing was found to be satisfactory. Further, followups were done at 7 and 18 months, respectively (Fig. 7).

\section{Discussion}

The primary aim of root canal treatment is to remove the pulpal tissue, bacteria, and their byproducts from the root canal space thus to cure or prevent periapical disease to promote the long-term survival of the tooth. In cases of the persisting disease, treatment options should include non-surgical retreatment and periapical surgery.

In an endodontically treated tooth, untreated canals could be due to the complexities in canal configuration, procedural errors, or the operator's limited knowledge of tooth anatomy. Missed canals serve as a reservoir of microorganisms and can have a negative impact on the treatment outcomes. In an unsuccessful endodontic treatment, signs and symptoms can vary from asymptomatic to an acute apical abscess. Conventional radiographs possess certain

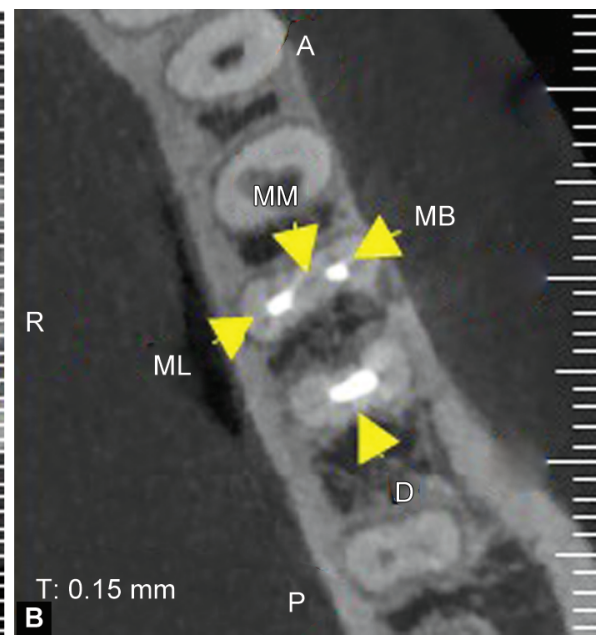


limitations when used to identify missed canals, due to their two-dimensional nature. With the advent of CBCT, it is possible to visualize the root canal anatomy three-dimensionally. Thus, it provides a more reliable and effective method for identification

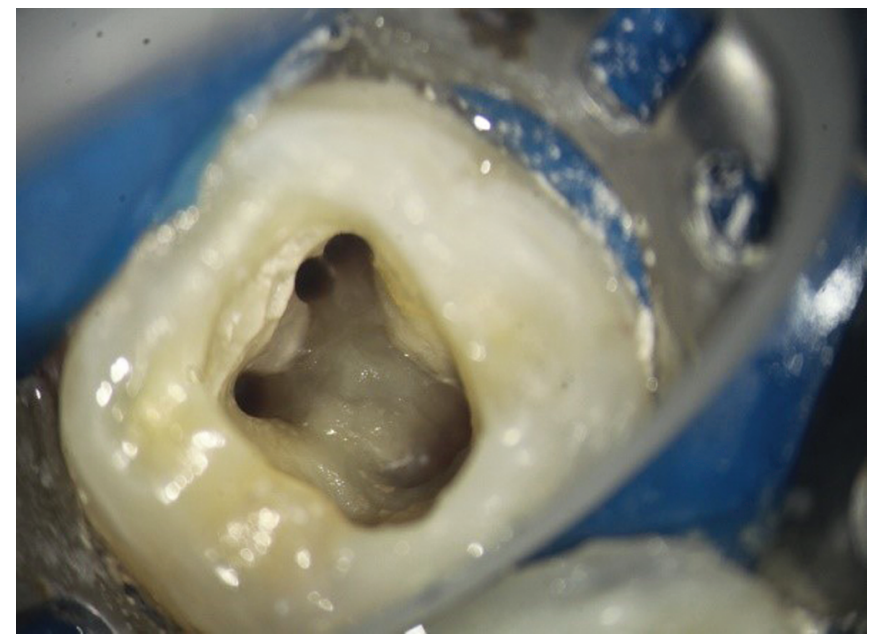

Fig. 3:View under the dental operating microscope shows canal orifices

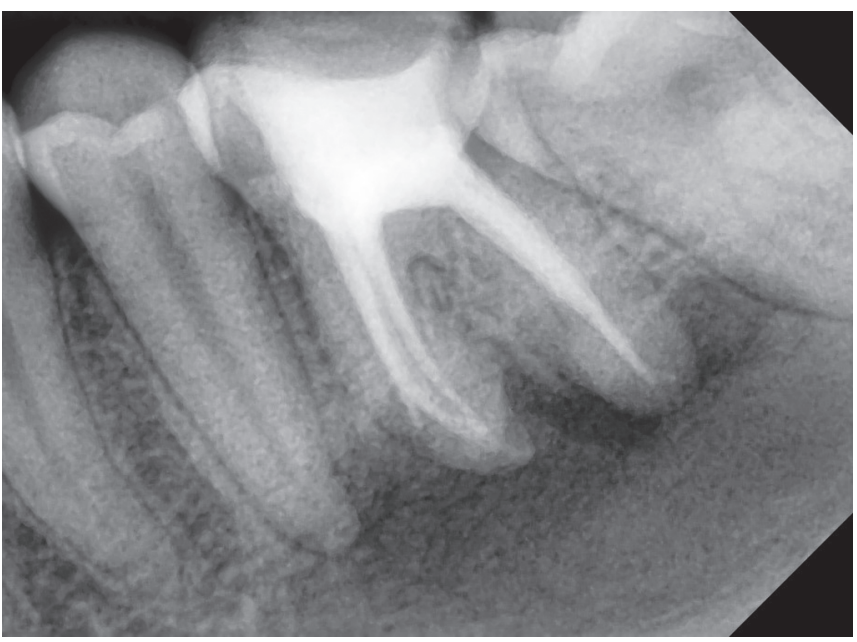

Fig. 5: Radiograph shows obturated canals
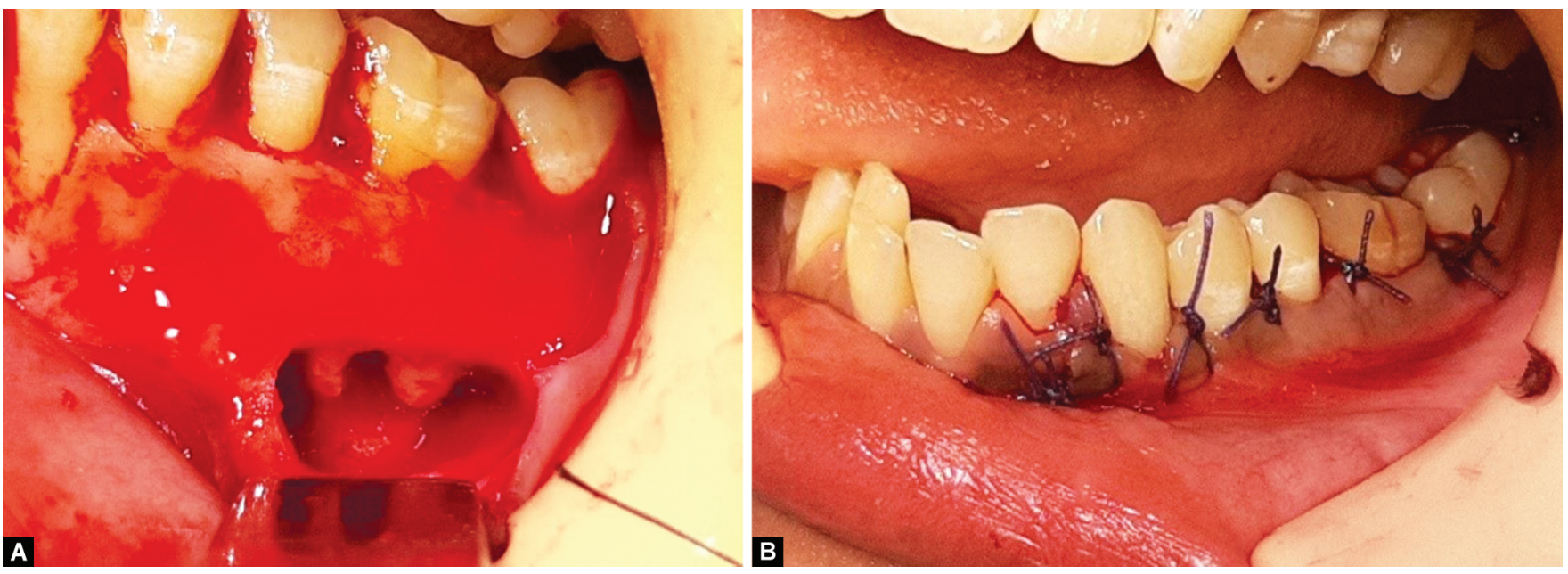

of the missed anatomy, accessory canals as well as the true nature and extension of the periapical lesions. Cone-beam computed tomography helps the clinician to preoperatively assess the entire surgical field without restriction, facilitating the surgical procedure.

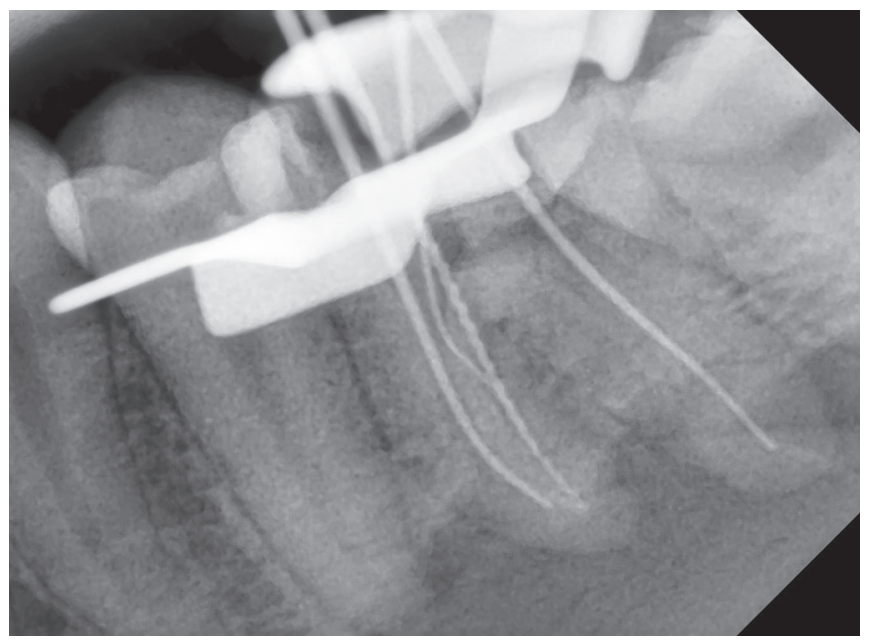

Fig. 4: Radiograph shows working length determination

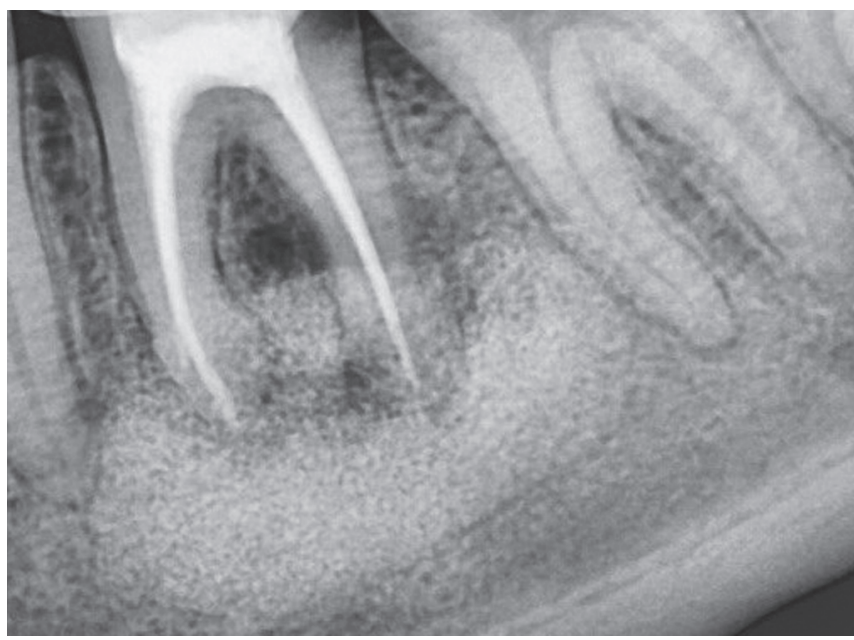

Fig. 7: 18 months review

Figs 6A and B: (A) Periapical surgery of 36; (B) Periapical surgery of 36 
The presence of middle mesial canals has been reported to be $15-45 \% .^{3}$ It was found more in the case of mandibular first molars compared with second molars. The prevalence was higher in younger patients aged between 20 years and 40 years. ${ }^{4}$ There is a definite relationship between ethnicity differences and the presence of middle mesial canals. Also, in vivo studies have reported higher prevalences than in vitro studies. ${ }^{5}$ Only $10-18 \%$ of the middle mesial canals were true mesial canals and $60-70 \%$ were anastomosis. ${ }^{4}$ Underfilled, unfilled, and missed canals are the common reasons for the failure of root canal procedures in mandibular molars. ${ }^{6}$ Therefore, techniques for locating and managing the middle mesial canals are of paramount importance for a successful outcome.

Among all the innovations, the introduction of operating microscopes together with the powerful ultrasonic units and instruments for microendodontics are considered to be the most important. The use of an operating microscope increased the accuracy of the middle mesial canal identification, especially when combined with ultrasonic troughing. ${ }^{7}$

Proper debridement of the root canal system is a challenging task, especially in cases with complex anatomies and retreatments. The use of good aseptic techniques, increased apical preparation sizes, the inclusion of full strength sodium hypochlorite, and $2 \%$ chlorhexidine irrigants are the most effective methods to eliminate E. faecalis. ${ }^{8}$ Surgical intervention compared to non-surgical root canal treatment reduces the time period required for healing of the lesions. Also in the case of chronic lesions and extraradicular infections, surgical treatment was found to be better.

On follow-up visits ( 7 and 18 months), the healing was found to be in progress in relation to tooth 36 and a crown was advised as a definitive restoration. Long-term follow-up is an important factor in assessing the treated tooth with a periapical lesion. Clinical as well as radiographic data are beneficial in monitoring the cases.

\section{Conclusion}

Missed canals have a significant impact on treatment prognosis. Compared with periapical radiographs, CBCT can provide a clear-cut view of the lesion size, extension, and missed canals as well. Magnification combined with ultrasonics is a valuable tool in locating the missed canals. While considering the retreatment of a tooth with a persisting lesion, a non-surgical approach should be the first treatment option followed by a periapical surgery especially in the case of chronic lesions.

\section{References}

1. Baruwa AO, Martins JNR, Meirinhos J, et al. The influence of missed canals on the prevalence of periapical lesions in endodontically treated teeth: a cross-sectional study. J Endod 2020;46(1):34-39.e1. DOI: 10.1016/j.joen.2019.10.007.

2. Basrani B, Tjäderhane L, Santos JM. Efficacy of chlorhexidine and calcium hydroxide containing medicaments against Enterococcus faecalis in vitro. Oral Surg 2003;96(5):618 De Pablo OV, Estevez R, Peix Sanchez M, et al. Root anatomy and canal configuration of the permanent mandibular first molar: a systematic review. J Endod 2010;36:1919-1931. DOI: 10.1016/s1079-2104(03)00166-5.

3. Nosrat A, Deschenes RJ, Tordik PA, et al. Middle mesial canals in mandibular molars: incidence and related factors. J Endod 2015;41(1):28-32. DOI: 10.1016/j.joen.2014.08.004.

4. Tahmasbi M, Jalali P, Nair MK, et al. Prevalence of middle mesial canals and isthmi in the mesial root of mandibular molars: an in vivo conebeam computed tomographic study. J Endod 2017;43(7):1080-1083. DOI: 10.1016/j.joen.2017.02.008.

5. Iqbal A. The factors responsible for endodontic treatment failure in the permanent dentitions of the patients reported to the college of dentistry, the University of Alijouf, Kingdom of Saudi Arabia. J Clin Diagn Res 2016;10(5):146-148. DOI: 10.7860/JCDR/2016/14272. 7884.

6. Mendes EB, Soares AJ, Martins JNR, et al. Influence of access cavity design and use of operating microscope and ultrasonic troughing to detect middle mesial canals in extracted mandibular first molars. Int Endod J 2020;53(10):1430-1437. DOI: 10.1111/iej.13352.

7. Stuart $\mathrm{CH}$, Schwartz SA, Beeson TJ, et al. Enterococcus faecalis: its role in root canal treatment failure and current concepts in retreatment. J Endod 2006;32(2):93-98. DOI: 10.1016/j.joen.2005.10.049.

8. Calişkan MK. Prognosis of large cyst-like periapical lesions following nonsurgical root canal treatment: a clinical review. Int Endod J 2004;37(6):408-416. DOI: 10.1111/j.1365-2591.2004.00809.x. 